\title{
On the Problem Orientation in the Evolution Process of Enterprise Strategic Management Theory-—Taking "Enterprise Competitive Advantage" as the Entry Point
}

\author{
Jing Zhang \\ School of Economics and Management, North China University of Technology, Beijing, China \\ Email: zhanjing_up@163.com
}

\begin{abstract}
The literature on the evolution of strategic management theory is vast, and the related literature is constantly increasing, and the theory is constantly being developed and improved. Many emerged strategic management schools and characteristic theories have provided profound insights on strategic management from different perspectives, different orientations and different trends. As stated in Mintzberg's book, "Our understanding of the formation of strategy is like blind people, no one has the vision of the whole elephant." Therefore, this paper attempts to analyze the many related issues with "enterprise competitive advantage" as the core. Based on the theory of strategic management, this paper analyzes the source of competitive advantage of enterprises and how to obtain sustainable competitive advantage based on the "problem-oriented" research ideas, in order to give a more reasonable and comprehensive explanation of the source of sustainable competitive advantage.
\end{abstract}

Keywords: Strategic management, corporate competitive advantage, resource-based theory.

\section{论企业战略管理理论演进过程中的问题导向性 一以 “企业竞争优势” 为切入点}

\author{
詹景 \\ 北方工业大学经济管理学院, 北京 \\ Email: zhanjing_up@163.com
}

\begin{abstract}
摘要：关于战略管理理论演进的文献浩如烟海，而且相关文献还在不断增加，理论也在不断的得到 发展和完善。涌现出的诸多战略管理学派和特色理论从不同视角、不同定位和不同趋势对战略管理 提出了深刻的见解。正如明茨伯格的书中所说的那样 “我们对战略形成的认识就如同盲人摸象, 没 有人具有审视整个大象的眼光”，所以本文试图以 “企业竞争优势” 为核心，在分析诸多相关战略 管理理论的基础之上, 以 “问题导向型” 研究思路, 对企业的竞争优势的来源以及如何获得持续竞 争优势等加以分析, 以期给出持续竞争优势来源的较合理、全面的解释。
\end{abstract}

关键词：战略管理，企业竞争优势，资源基础理论

\section{1 前言}

从战略管理理论的形成与发展历程以及企业战略管理实践的演进过程看, 战略管理无疑是充满生命力的 系统过程。在理论的演进过程中, 我们始终关注着 “如何制定战略、谁制定战略、由谁怎样制定什么样 的战略”一系列问题, 在问题发现与解决后, 又对其进行概括、总结进而形成理论体系; 企业在谋求生 存、成长、发展、壮大的进程中面临的战略问题层出不穷, 无外乎如何根据环境的变化不断调整、更 新、优化自身条件以获得先进于竞争对手的持续优势或与其他企业组成战略联盟, 实现合作共赢而获取 超额收益, 继而基业长青。但是, 将所有问题归纳、总结于此, 反而不利于我们发现和解决问题, 所以 本文选择的问题切入点为 “企业竞争优势” 。尽管现在所有的战略管理理论, 如竞争理论、资源基础理 
论、能力理论、知识管理理论、动态竞争理论等, 均对企业如何获取竞争优势问题有所解释, 然而, 理 论导向型的学术逻辑思维往往缺乏系统性、完整性（张广才，2004）。马克思在《莱茵报》第 137 号刊 论《集权问题》中提出: 历史本身除了通过提出新问题来解答和处理老问题之外, 没有别的方法。树立 问题意识、坚持问题导向, 是新的时代条件下开创事业发展新局面的必然要求（刘云山, 2014）。因 此, 以 “企业竞争优势” 为问题导向, 对相关理论进行探讨, 并围绕企业在生存、发展、成长、壮大过 程中面临的主要问题——企业的竞争优势是什么、如何获取企业竞争优势以及持续竞争优势的获得, 将 各个理论加以梳理、整合，对 “企业竞争优势” 相关问题进行系统阐释，并展望未来和提出对应的建 议，将对企业战略管理理论和战略管理实践的改进有所禆益。

战略管理理论的形成与发展大致可以分为萌芽阶段（1900-1950）、形成阶段（1950-1980）、发展阶 段（1980-至今）（亨利・明茨伯格，2012）。事实上，对于战略管理理论研究的划分目前并没有统一 的标准。尽管如此, 亨利 - 明茨伯格将战略管理理论划分为十大学派, 并系统梳理每个学派的思想, 介 绍与战略有关的基本概念, 而且在描述和点评每个学派的观点时, 剖析其贡献和局限, 最后为读者呈现 一头完整的战略管理大象和一幅横跨半个多世纪的战略发展全景图。至今, 我们发现不管关于战略管理 的研究文献浩如烟海, 不管理论如何推陈出新, 都能在明茨伯格所划分的十大派别中找到其踪影。这也 意味着多种学派综合体的出现, 管理实践日趋复杂。本文试图在分析诸多战略管理理论的基础上, 重点 结合资源基础理论的演进，提出 “问题导向型”企业竞争优势模型。

\section{2 理论述评}

\section{1 企业外部环境与竞争优势}

20 世纪 60 年代初产生的设计学派, 无疑是战略形成过程中最具影响力的学派之一。该学派主要在塞兹 尼克 (Selznick) 、钱德勒 (Chandler)、安德鲁斯 (Andrews) 等人的推动下产生, 该学派的核心目标 是建立内部能力与外部环境的匹配（亨利・明茨伯格, 2012）。“经济战略就是在企业所处环境中能够 决定其地位的机遇与限定条件之间的匹配” (Christensen, 1982) , 是该学派最著名的学者的话。由此 可以看出设计学派观点下, 竞争优势来源于企业内部优势、劣势与外部环境中的威胁、机会的匹配, 企 业应该在已经确定的活动领域内, 根据环境的状况及内部条件建立和保持竞争优势（李萍, 李刚亨利, 2008）。但是, 设计学派的适用于相对稳定或者至少可以预测的环境, 而且战略的制定与执行是分离 的，尽管它在表述战略方面颇有贡献。

\section{2 产业结构和企业竞争优势}

从以美国战略管理学家迈克尔 - 波特 (M.E.Porter) 为代表的竞争战略理论中可以得出, 企业的竞争优 势源于产业结构和企业在所属产业的吸引力和市场地位 (马刚, 2006)。波特提出在一个产业中存在潜 在进入者的威胁、替代品的威胁、供应商议价能力、购买者议价能力以及现有企业间的竞争五种基本竞 争力量, 这五种力量的状况及综合强度, 引起产业内的经济结构的变化。而产业竞争结构决定着产业内 部竞争的程度和产业盈利的能力（亨利 - 明茨伯格, 2012)。所以, 企业如果想要使自己拥有长期竞争 优势, 必须使得企业所处的竞争结构是有利的。通过五力竞争模型的分析, 确定企业在市场环境中的地 位和所处的产业吸引力, 进而根据现实的竞争格局, 只要采取成本领先和差异化战略获取竞争优势。 后来, 波特还引入了价值链分析工具, 试图从企业内部价值创造过程中寻求企业的竞争优势, （王革, 吴练达, 张亚辉, 2004) 以便于企业实施基本的竞争战略。综合看波特的竞争战略理论, 其理论贡献我 们不能否认, 但是其战略只是告诉企业在何种产业结构下采用何种战略, 至于如何改变市场结构、现实 格局, 却没有给出具体的引导。而且, 竞争战略理论相较于企业自身内部条件, 更多关注的是产业环 境、市场竞争结构, 对处于无吸引力产业的企业获得高利润、盈利水平很高的行业也会存在经营业绩很 差等现象的解释却无能为力。当人们逐渐意识到这些问题时, 以企业如何获取并维持竞争优势为核心问 题的企业资源基础理论研究便得以陆续开展。

\section{3 企业内部条件与竞争优势}

为了更好地理解与探求企业是如何获取并维持竞争优势的, 20 世纪 80 年代中期出现的 (1984 年沃纳菲 尔特的 “企业的资源基础论” 的发表意味着资源论的诞生）（王革, 吴练达, 张亚辉，2004）资源基础 理论将战略管理研究的主流视角——关注产业和竞争环境的外部分析, 转向关注企业内部的异质性资源 和能力, 并认为企业竞争优势来源于组织的资源（吴金南，刘林，2011）。近些年来，资源基础理论已 经成为理解企业竞争优势的重要理论依据, 并在理论界和实践领域产生广泛的影响。可是, 资源基础理 
论研究者对企业资源的界定不一致且该理论学派众多, 导致至今仍没有比较统一的理论体系的研究分析 范式, 也不利于从问题导向研究此理论的演变与发展。因此, 接下来本文尝试以 “获取和维持竞争优 势”问题导向为主线，对企业资源基础理论的演变过程进行分析与评价。

\subsection{1 传统的资源基础理论}

企业资源基础理论的贡献在于其将主流的对企业竞争优势的理论研究从产业结构等外部因素转为企业 内部所拥有的异质性资源。该理论的核心观点是, 企业是由一系列资源束组成的集合, 企业的竞争优势 源自于企业所拥有的资源, 尤其是一些异质性资源, 外部的市场结构与市场机会对企业的竞争优势产生 一些影响，但并不是决定因素（杨春华，2008）。由于对 “资源” 的界定不同，所以传统的资源基础观 把企业竞争优势界定在具体的作为物的资源上（杨春华，2008）, 并以企业单位为分析对象, 研究、分 析企业所拥有的各项内部资源, 并以此为切入点, 寻求企业的异质性资源和独特能力, 以获取和维持企 业的竞争优势。传统的资源基础观中企业资源具有 “异质性(heterogeneity)”，在企业间具有 “非完全 流动性”（吴金南, 刘林, 2011）。因此, 有价值的、稀缺的、难以模仿和不可替代的资源是企业获得 和维持竞争优势的源泉。

\subsection{2 企业能力理论}

企业所拥有的异质性资源, 如厂房、设备、资金等, 的确可以使一个企业在向顾客提供有价值的产品 或者服务的过程中表现出较于竞争对手的优势而具有更强的盈利能力。但是, 传统的资源基础观将资源 局限在企业所拥有的具体物上，而对企业中的人关注不多。事实上，企业中的人是企业资源的配置者， 如果仅仅存在客观的物质资源, 而没有发挥人的能动性, 那么企业异质性的资源往往会得不到充分的利 用。这就如同棋盘上的一盘棋, 棋子和棋局已如此, 关键是下棋的人有能力将棋局看透并能与对方斗智 斗勇而胜其半子。因此, 研究者对传统资源基础观进行 “扬弃” 后, 提出了企业能力理论, 其标志是论 文《The core competence of the corporation》（Prahalad、Hamel, 1990）。但并不是所有的能力都能 形成企业竞争优势, 唯有核心能力才能成为竞争优势的来源, 这即是核心能力理论的观点 (Prahalad C K, Hamel G，1990）。尽管基础能力理论（Sanchez、Heene，1997）认为企业应当把能力视作一个开 放的系统来管理, 但是限于现实的条件, 企业不可能关注到企业的方方面面, 而是应该抓主要矛盾, 坚 持问题导向, 而解决企业所面临的挑战和问题。因此, 核心能力的挖掘、培育、积累、维持和正确地运 用才是企业获得和维持企业竞争优势的来源。虽然如此, 但是核心能力的维持却不是那么容易维持的, 尤其在当今复杂变幻的市场竞争环境下，具备相应的种种基础能力也是必要的。

\subsection{3 知识基础理论}

在核心能力理论和组织学习理论之上发展的知识基础理论, 试图探讨企业核心能力的决定因素。虽不 知将此理论纳入战略管理理论范畴是否合适, 因为其涉及企业理论的一些核心问题, 但是它的存在也确 实能解释一些问题。企业竞争优势的获取和维持源于企业所具有的核心能力, 但是核心能力的背后是企 业掌握的知识, 尤其是隐性知识, 正是这些异质性知识才是企业竞争优势的源泉。基础知识理论认为不 同状况的企业产生的隐形知识，会在企业个人身上和实践、经验中得到表现，进而有利于企业竞争优势 的获取和维持 (Conner K R, Prahalad C K, 1996) 。当然, 如果要进一步探寻, 会得出企业知识的 获取是通过人的学习而获得的, 而且还需要知识管理, 在此便不再赘述。

\subsection{4 核心能力理论}

不管是传统的资源基础观, 还是核心能力理论或者知识基础理论, 都是以静态的视角探讨企业竞争优 势的来源和决定性因素, 而鲜有从动态角度做出解释的。可是, 日益变化的企业外部环境使企业面临更 多的挑战和机遇。特别是处于 “超竞争” 的环境下, 竞争优势难以持续, 不仅因为能力存在惯性, 而且 竞争对手可以借助有利渠道在此环境背景下迅速模仿管理技巧、新技术、商业模式、加大资本投入等来 迎合和满足顾客的及时需要 (马刚, 2006)。所以, 如何在这种复杂多变的竞争环境中对企业内部资源 进行动态调整、降低资源刚性和核心刚性以获得并保持竞争优势（姚小涛，2003），便是战略资源研究 者的又一个重要的研究内容。资源基础观的继承与发扬者提斯（David J. Teece）, 在研究过程中不再 仅是强调企业现有资源与能力存量的多少, 而是引入了动态因素, 突出对 “动态能力” 的关注（谭力 文, 丁靖坤, 2014) 。认为从静态角度研究企业竞争优势是有限的, 所以动态竞争理论提出企业应具备 “企业整合、建立和在配置内外部能力以适应快速变化环境的能力” 的动态能力, 并且强调管理者要关 注企业内部过程, 以增强技能、胜任和特定资产为战略基点, 进而决定企业能力的发展方向, 推动企业 的学习（宋波,徐飞, 伍青生, 2011) 。近些年来, 动态能力理论的研究显然是战略研究者关注的热 点, 对于动态能力的概念界定、外在特征、内在机理和影响因素、与竞争优势的关联性、贡献与不足以 及展望, 都进行了细致地研究和探讨。动态能力理论已然成为较成熟的战略管理理论。虽然对其与竞争 优势的关联仍然有所分歧, 但是本文认为动态能力是企业持续竞争优势的来源, 而在动态能力调整配置 下的资源与能力是企业竞争优势的来源, 这样也符合企业动态能力理论对静态研究企业竞争优势的突 破。当然, 动态能力理论也具有其局限性, 对于动态能力的测量或者评价维度也有待进一步研究, 但是 它依然具有很重要的理论和实践意义。 


\section{3 理论模型}

我们对资源基础理论进行分析与评述, 并基于 “企业竞争优势” 问题导向相继引入了设计学派、竞争战 略理论以及企业资源基础理论, 而且对于资源基础观, 我们探讨了核心能力、知识、动态能力等关键 词, 从外部环境到企业内部资源与能力, 从静态视角到动态视角研究, 这都体现了我们的战略管理越来 越富有柔性, 并为竞争到超竞争环境的转变而做出动态调整, 以适应复杂多变的市场环境而获取持续竞 争优势。关于企业竞争优势的来源，本文将其归纳如图 1。

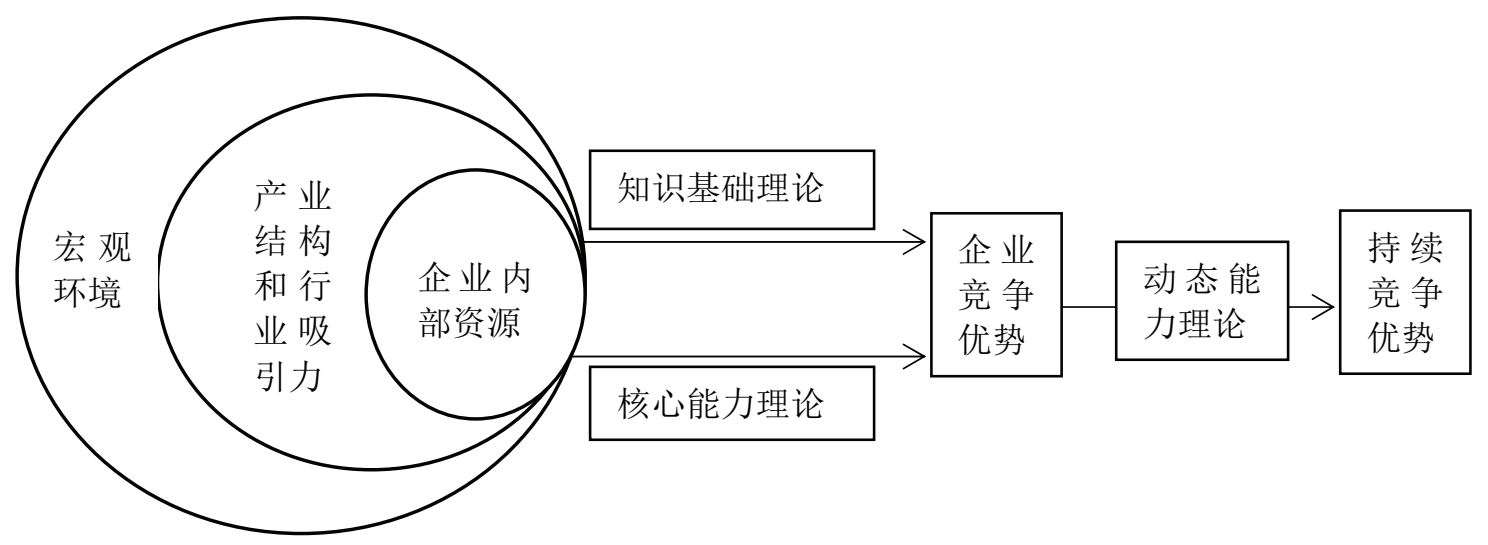

图 1. 企业竞争优势来源的推演模型

从图 1 , 我们可以看出企业要想获得持续竞争优势, 就需要具备一定的能力, 且这种能力是动态的, 是与时俱进的, 而不是一成不变的。而能力与知识的获得与企业内部环境、宏观环境以及产业结构和行 业吸引力有关。因此, 处于一日千里的社会背景及激烈的市场竞争环境下的企业需要动态能力, 更需要 常常洞察企业内部环境、宏观环境以及产业结构和行业吸引力, 从文化、产业、资本、人力、运营、管 理、战略等方面运筹帷幄, 打下坚实的基础, 方能以不变应万变, 得以基业长青。

\section{4 总结}

未来对于动态能力观的研究, 不仅应关注动态能力的培养、测量和完善, 也应该结合企业外部环境, 根 据企业现实情况进行动态调整。而且, 在目前这样复杂多变的环境中, 企业不能只是谋求比竞争对手拥 有优势, 也可以寻求企业间的合作与联盟从而实现共赢。而且, 我们发现, 在战略的制定与实施过程 中, “人” 作为不可或缺的角色发挥着主观能动性。归根结底, 人才是企业持续竞争优势的重要来源。 战略管理与人力资源管理的深度融合是我们值得期待的。

斯图尔特 - 克雷纳在《管理百年》中说 “管理学总是挑战那些试图将它引入角落, 使它固定下来的理 论家。它总是能从这些理论家手里逃脱。它也总是能从他们的指缝里逃走”。我想这或许就是管理学的 魅力所在和有趣之处。对管理信息进行清晰的全面描述, 我们可能做不到这点, 但是我们可以采用问题 导向意识来研究它。坚持问题导向意识, 当管理实践出现问题时, 运用相关理论指导实践、解决问题。 如果已有理论不可行, 那就在寻求新的解决办法的过程中发展它、完善它。这样或许会存在滞后性, 但 是往往出色的战略家不乏远见和对未来可能发生的不确定性问题的预测能力。

\section{参考文献}

1. 张广才, “战略收益” 导向的战略观阐释 [J], 外国经济与管理, 2004 .

2. 亨利 - 明茨伯格, 战略历程: 穿越战略管理旷野的指南（原书第 2 版） [M], 机械工业出版社, 2012.

3. 李萍, 李刚, 企业竞争优势根源的理论述评 $[\mathrm{J}]$, 重庆工商大学学报（社会科学版）, 2008.

4. 马刚, 企业竞争优势的内涵界定及其相关理论评述 $[\mathrm{J}]$, 经济评论, 2006.

5. 王革, 吴练达, 张亚辉, 企业战略管理理论演进与展望 $[\mathrm{J}]$, 科学学与科学技术管理, 2004.

6. 吴金南, 刘林, 国外企业资源基础理论研究综述 $[\mathrm{J}]$, 安徽工业大学学报（社会科学版）, 2011.

7. 杨春华, 资源概念界定与资源基础理论述评 $[\mathrm{J}]$, 科技管理研究, 2008 . 
8. Prahalad C K, Hamel G, The Core Competence of the Corporation, Harvard Business Review, 1990, 68 (3): 79 72 .

9. Conner K R, Prahalad C K, A Resource-based Theory of the Firm: Knowledge Versus Opportunism. Organization Science, 1996, 7 (5): 477-501.

10. 姚小涛, 战略管理理论研究的发展历程与展望 $[\mathrm{J}]$, 预测, 2003.

11. 谭力文, 丁靖坤, 21 世纪以来战略管理理论的前沿与演进: 基于 SMJ (2001-2012) 文献的科学计量分析 [J], 南开管理评论，2014，17（2）：84-94.

12. 宋波, 徐飞, 伍青生, 企业战略管理理论研究的若干前沿问题 $[J]$, 上海管理科学, 2011 . 